\title{
ADSL Transceivers Applying DSM and Their Nonstationary Noise Robustness
}

\author{
Etienne Van den Bogaert, ${ }^{1}$ Tom Bostoen, ${ }^{2}$ Jan Verlinden, ${ }^{2}$ Raphael Cendrillon, ${ }^{3}$ and Marc Moonen ${ }^{4}$ \\ ${ }^{1}$ Research \& Innovation Department of Alcatel, Francis Wellesplein 1, 2018 Antwerpen, Belgium \\ ${ }^{2}$ Access Networks Division of Alcatel, Francis Wellesplein 1, 2018 Antwerpen, Belgium \\ ${ }^{3}$ School of Information Technology \& Electrical Engineering, University of Queensland, Brisbane, QLD 4072, Australia \\ ${ }^{4}$ Department of Electrical Engineering, Katholieke Universiteit Leuven, Kasteelpark Arenberg 10, 3001 Leuven-Heverlee, Belgium
}

Received 10 December 2004; Revised 10 May 2005; Accepted 18 May 2005

Dynamic spectrum management (DSM) comprises a new set of techniques for multiuser power allocation and/or detection in digital subscriber line (DSL) networks. At the Alcatel Research and Innovation Labs, we have recently developed a DSM test bed, which allows the performance of DSM algorithms to be evaluated in practice. With this test bed, we have evaluated the performance of a DSM level-1 algorithm known as iterative water-filling in an ADSL scenario. This paper describes the results of, on the one hand, the performance gains achieved with iterative water-filling, and, on the other hand, the nonstationary noise robustness of DSM-enabled ADSL modems. It will be shown that DSM trades off nonstationary noise robustness for performance improvements. A new bit swap procedure is then introduced to increase the noise robustness when applying DSM.

Copyright (C) 2006 Hindawi Publishing Corporation. All rights reserved.

\section{INTRODUCTION}

DSL deployment is evolving to, on the one hand, ever higher bit rates enabling video services over DSL, and, on the other hand, increased reach to enlarge the customer base. Higher bit rates as well as increased reach can either be obtained by deploying remote terminals (RTs) or by applying dynamic spectrum management (DSM) techniques $[1,2]$. The latter technology can provide rate/reach improvements on the shorter term, because it only requires software adaptations, whereas RT deployment involves heavy investments, and hence is rather suited for the longer term.

DSM is an adaptive form of spectrum management [3] and is based on automatic detection of interference caused by crosstalk. From this perspective, the entire twisted-pair binder is considered as a shared resource and the overall bit rate is optimized. This optimization can be done in different ways, depending on the level of coordination between the multiple DSL lines. We remark that the name "dynamic spectrum management" originates from adaptive multiuser power allocation techniques, but the meaning of the term DSM has widened to include also multiuser detection techniques.

A distinction is made between DSM at levels $0,1,2$, and 3 according to the degree of coordination. Level-0 DSM means there is no coordination between the lines. DSM at level 1 means that the bit rates are reported to and controlled by a spectrum management centre (SMC). The actual transmit PSDs are computed in each transceiver, hence the multiuser power control is distributed. At level 2, the received signal and noise power spectral densities (PSDs) are reported to the SMC and the transmit PSDs are controlled by the SMC [4]. Both level 1 and 2 gains in rate and reach are originating from adaptive multiuser power allocation techniques, resulting in crosstalk avoidance. Finally, level 3 is the highest DSM level at which all colocated transceivers jointly process the received symbols for upstream transmission and the transmit symbols for downstream transmission [5]. At this level, the gains are originating from multiuser detection techniques based on either crosstalk cancellation or crosstalk precompensation.

In this paper, we concentrate on DSM at level 1, and in particular on a specific DSM algorithm called iterative waterfilling [2], as well as a simplified version thereof. In Sections 2 and 3, we first review DSL channel properties and distributed multiuser power allocation before detailing the practical implementation of iterative water-filling on DSL modems. In Section 4, the real-life performance of iterative water-filling is demonstrated in an ADSL scenario, showing data-rate gains of up to $500 \%$ in realistic settings. Finally, in Section 5, some questions are raised about DSM trading off nonstationary noise robustness for performance. The nonstationary noise robustness is further investigated and a new 
bit swap procedure for enhanced noise robustness is proposed showing substantial improvements.

\section{THE DSL CHANNEL MODEL AND BIT LOADING}

We focus on DSL modems using discrete multitone (DMT) modulation, as for example, adopted in the ADSL standard [6]. The bit loading is calculated on a per-tone basis, as given by (1) for a two-user case, and depends on the signal-to-noise ratio $(\mathrm{SNR})$ at the receiver:

$$
\begin{aligned}
b_{k}^{1} & =\log _{2}\left(1+\frac{\mathrm{SNR}_{1}(k)}{\Gamma_{1}}\right) \\
& =\log _{2}\left(1+\frac{S_{1}(k) \cdot h_{11}^{2}(k)}{\Gamma_{1}\left(N_{1}(k)+S_{2}(k) \cdot h_{12}^{2}(k)\right)}\right) .
\end{aligned}
$$

In (1), $k$ represents the tone index, $N_{1}(k)$ denotes all the noises other than self-crosstalk, and $\Gamma_{1} \approx 12 \mathrm{~dB}$ is equal to the SNR gap including noise margin and coding gain. The SNR gap to achieve a bit error rate (BER) of $10^{-7}$ is approximately equal to $9.75 \mathrm{~dB}$. Adding to this a noise margin of $6 \mathrm{~dB}$ minus a coding gain of $3.75 \mathrm{~dB}$, one obtains an overall value of $12 \mathrm{~dB}$ for $\Gamma_{1} . S_{i}(k)$ denotes the transmit PSD of user $i$ on tone $k, h_{11}(k)$ represents the direct channel transfer function of user 1 and $h_{12}(k)$ denotes the crosstalk channel transfer function from user 2 to user 1 .

The bit loading given by (1) allows the modem to adapt to the changing line conditions by dynamically varying the constellation used on each tone. Moreover, (1) tells us that the bit loading for user 1 depends on the crosstalk coming from the other users. If the crosstalk increases on a particular carrier, fewer bits can be put on this carrier. The same is true for the other users, where the crosstalk coming from user 1 interferes with the signal of the other users. To illustrate the importance of crosstalk, an example of measured channel transfer functions for a $1400 \mathrm{~m}$ section of a $0.4 \mathrm{~mm}$ 4-quad France Telecom cable is shown in Figure 1. The farend crosstalk (FEXT) will be, in this case, on average equal to $-120 \mathrm{dBm} / \mathrm{Hz}$, as the nominal transmit PSD of ADSL modems is equal to $-40 \mathrm{dBm} / \mathrm{Hz}$.

\section{MULTIUSER POWER ALLOCATION}

The goal of multiuser power allocation is to optimize the overall bit rate while all transceivers are also subject to a total power constraint. This constrained optimization problem is given by (2) for the two-user case:

$$
\max R_{S_{1}(k), S_{2}(k)} \text { s.t. }\left\{\begin{array}{l}
\sum_{k} S_{1}(k) \leq P_{1}, \\
\sum_{k} S_{2}(k) \leq P_{2},
\end{array}\right.
$$

with $R=\sum_{k} b_{k}^{1}+\sum_{k} b_{k}^{2}$, the rate sum.

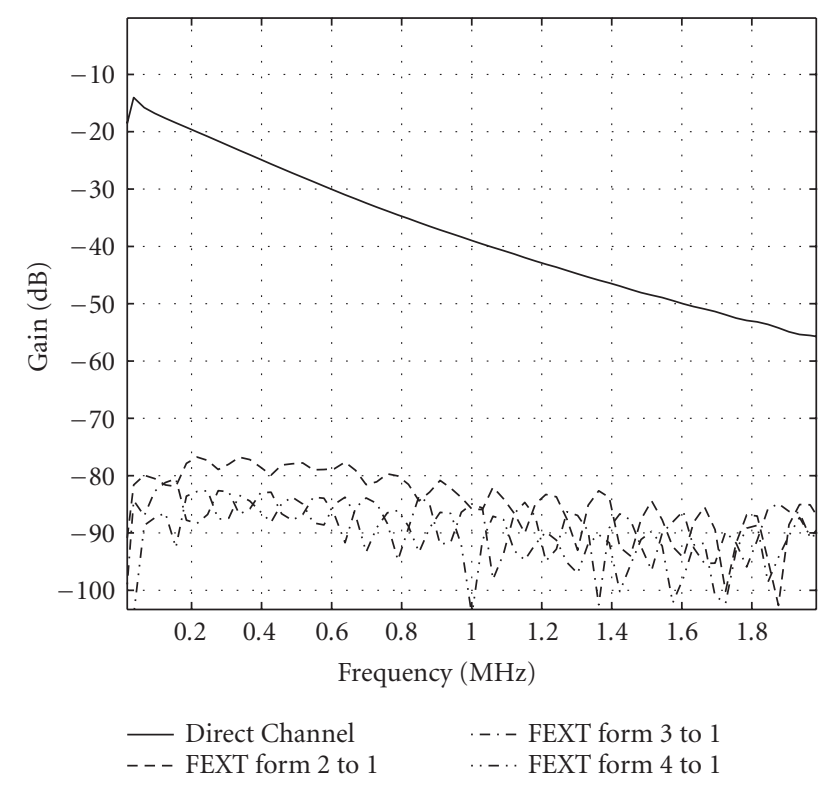

FIgURE 1: Direct and FEXT channel transfer functions of a $1400 \mathrm{~m}$ section of a 4 -quad $0.4 \mathrm{~mm}$ France Telecom cable.

This constrained optimization problem can be solved by means of the Lagrangian, which is equal to

$$
\begin{aligned}
J\left(S_{1}(k), S_{2}(k)\right) & \\
= & \sum_{k} \log _{2}\left(1+\frac{S_{1}(k) \cdot h_{11}^{2}(k)}{\Gamma_{1}\left(N_{1}(k)+S_{2}(k) \cdot h_{12}^{2}(k)\right)}\right) \\
& +\sum_{k} \log _{2}\left(1+\frac{S_{2}(k) \cdot h_{22}^{2}(k)}{\Gamma_{2}\left(N_{2}(k)+S_{1}(k) \cdot h_{21}^{2}(k)\right)}\right) \\
& +\lambda_{1} \cdot\left(P_{1}-\sum_{k} S_{1}(k)\right)+\lambda_{2} \cdot\left(P_{2}-\sum_{k} S_{2}(k)\right) .
\end{aligned}
$$

Equation (3) is the sum of the bit rates of both users together with the Lagrange multipliers taking into account the total power constraint of both users. This is a non-convex optimization problem. Hence, finding an optimum requires an exponential complexity in $K$, with $K$ the total number of tones. In recent work [7], numerically tractable ways of solving this problem through use of a dual decomposition have been developed. Whereas this algorithm demonstrates large performance gains, it is centralized and requires the existence of a spectrum management centre (SMC). In this work, we focus on a distributed algorithm which does not require an SMC. This algorithm is known as iterative water-filling [2]. Iterative water-filling can be derived by first making the assumption that the crosstalk noise is temporarily constant and that it can be incorporated in the term representing the background noise. This results in a simplified Lagrangian, 


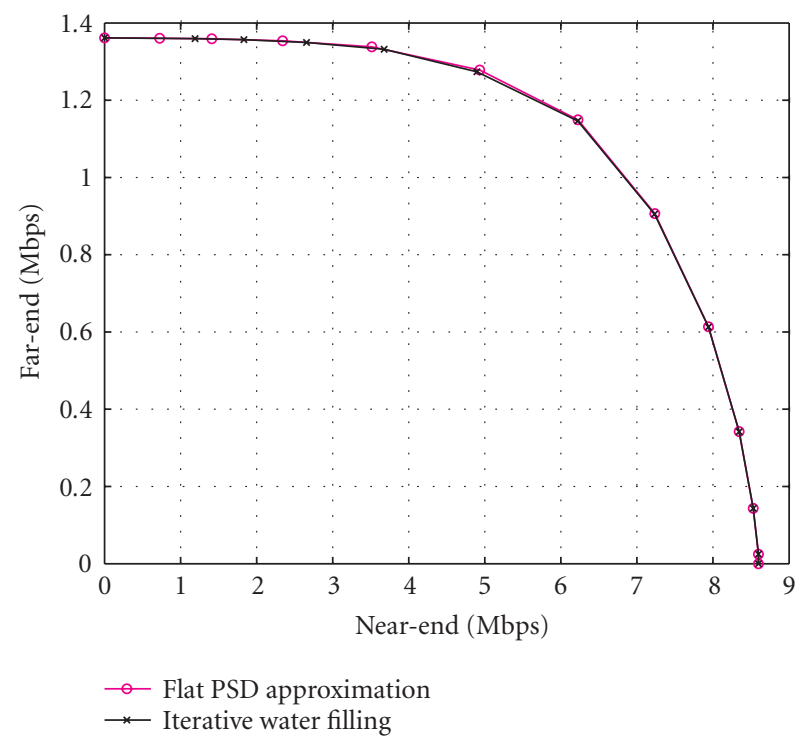

FIGURE 2: Iterative water-filling and flat PSD rate regions.

equation (4), with the optimum given by (5):

$$
\begin{aligned}
J\left(S_{1}(k), S_{2}(k)\right) & \\
= & \sum \log _{2}\left(1+\frac{S_{1}(k) \cdot h_{11}^{2}(k)}{\Gamma_{1} N_{1}(k)}\right) \\
& +\sum \log _{2}\left(1+\frac{S_{2}(k) \cdot h_{22}^{2}(k)}{\Gamma_{2} N_{2}(k)}\right) \\
& +\lambda_{1} \cdot\left(P_{1}-\sum_{k} S_{1}(k)\right)+\lambda_{2} \cdot\left(P_{2}-\sum_{k} S_{2}(k)\right), \\
S_{1}(k)= & {\left[\frac{1}{\lambda_{1} \ln 2}-\frac{\Gamma_{1} N_{1}(k)}{h_{11}^{2}(k)}\right]^{+}, } \\
S_{2}(k)= & {\left[\frac{1}{\lambda_{2} \ln 2}-\frac{\Gamma_{2} N_{2}(k)}{h_{22}^{2}(k)}\right]^{+}, }
\end{aligned}
$$

where $[x]^{+}=\max (0, x)$.

The iterative water-filling solution is then obtained by replacing the background noise with the total noise in (5), leading to

$$
\begin{aligned}
& S_{1}(k)=\left[\frac{1}{\lambda_{1} \ln 2}-\frac{\Gamma_{1}\left(N_{1}(k)+S_{2}(k) \cdot h_{12}^{2}(k)\right)}{h_{11}^{2}(k)}\right]^{+}, \\
& S_{2}(k)=\left[\frac{1}{\lambda_{2} \ln 2}-\frac{\Gamma_{2}\left(N_{2}(k)+S_{1}(k) \cdot h_{21}^{2}(k)\right)}{h_{22}^{2}(k)}\right]^{+} .
\end{aligned}
$$

Assuming the crosstalk noise to be constant is not valid when considering a larger time window. So, each time the crosstalk noise changes, the modems will adapt to this timevarying noise environment and adapt their transmit PSD. This means that there will be an iteration of modems applying water-filling, hence this explains the name "iterative water-filling." Applying these power allocation formulas iteratively is proved to converge to a so-called Nash equilibrium [2].
From (1), it follows that, to have one bit on a carrier, the SNR must be at least as large as $\Gamma_{1}$. Combining this with (6), the transmit PSD on tones loaded with 1 bit will be given by.

$$
S_{1}^{\min }(k)=\frac{\Gamma_{1}\left(N_{1}(k)+S_{2}(k) \cdot h_{12}^{2}(k)\right)}{h_{11}^{2}(k)}=\frac{1}{2 \lambda_{1} \ln 2} .
$$

On the other hand, the transmit PSD on tones with very low noise-to-channel ratio (NCR) will be approximated by (8). As a conclusion, the transmit PSD is seen to vary only with at most $3 \mathrm{~dB}$ :

$$
S_{1}(k)=\frac{1}{\lambda_{1} \ln 2}
$$

The water-filled transmit PSD can then be approximated by one PSD level for all usable tones, equal to the total power divided by the useful transmit bandwidth. The simplicity of this water-filling approximation decreases the power allocation complexity of DSM applied at level 1. Although the complexity of water-filling as such is not that high, this approximation has one clear advantage: existing ADSL implementations (which all use flat PSD allocation) can be used for DSM level 1 by just controlling their average PSD level. Figure 2 shows the rate regions of water-filling and the flat PSD approximation, respectively. As can be seen from the figure, the difference in performance is negligible. The simulation scenario is the same as the scenario shown in Figure 3, and which will be explained in the next section.

Note that the resulting iterative procedure is straightforwardly generalized to the $N$-user case.

Finally, an important aspect is that a DSL transceiver can be operated in 3 so-called adaptation modes. In rateadaptive (RA) mode, the transceiver uses all available power to maximize the bit rate, while maintaining a fixed noise margin. Similarly, in margin-adaptive (MA) mode, the transceiver uses all available power to maximize the noise margin, while maintaining a fixed bit rate. Finally, in power-adaptive (PA) mode, the transceiver minimizes the power consumption, while maintaining a fixed bit rate and noise margin. Currently, most DSL lines are operated in MA mode, which means that a lot of power is wasted on the short loops, also generating unnecessary crosstalk on the longer loops. DSM at level 1 proposes to switch all DSL transceivers to PA mode, this means that a DSL transceiver connected to a short loop will apply power back-off (PBO) in order to minimize its power. Furthermore, it is also proposed to abandon the idea of using spectral masks to ensure spectral compatibility with other DSL services, but only to restrict the total power. Hence, a DSL transceiver connected to a long loop would be allowed to reallocate power from the higher tones, which are then not used, to the lower tones, a technique called boosting.

\section{DSM PERFORMANCE}

Figure 3 shows a block diagram of the DSM (level 1) demonstrator at Alcatel Research and Innovation Labs, which has provided the results shown in Figures 4 and 5. The demonstrator is based on ADSL modems and a mixed deployment 


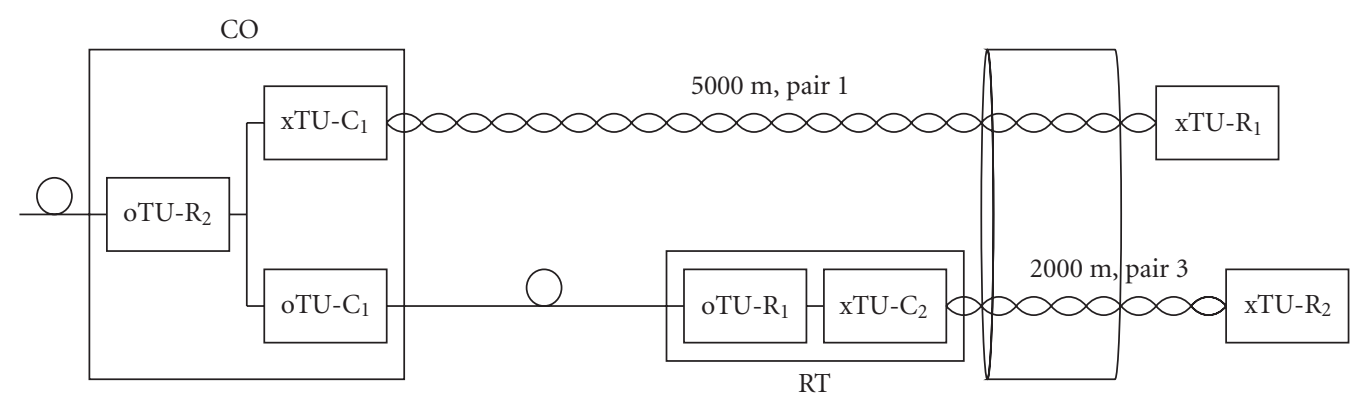

FIGURE 3: DSM demonstrator at Alcatel Research \& Innovation Labs implementing 1 long CO line of $5000 \mathrm{~m}, 1$ short RT line of $2000 \mathrm{~m}$, and a distance CO-RT of $3000 \mathrm{~m}$.

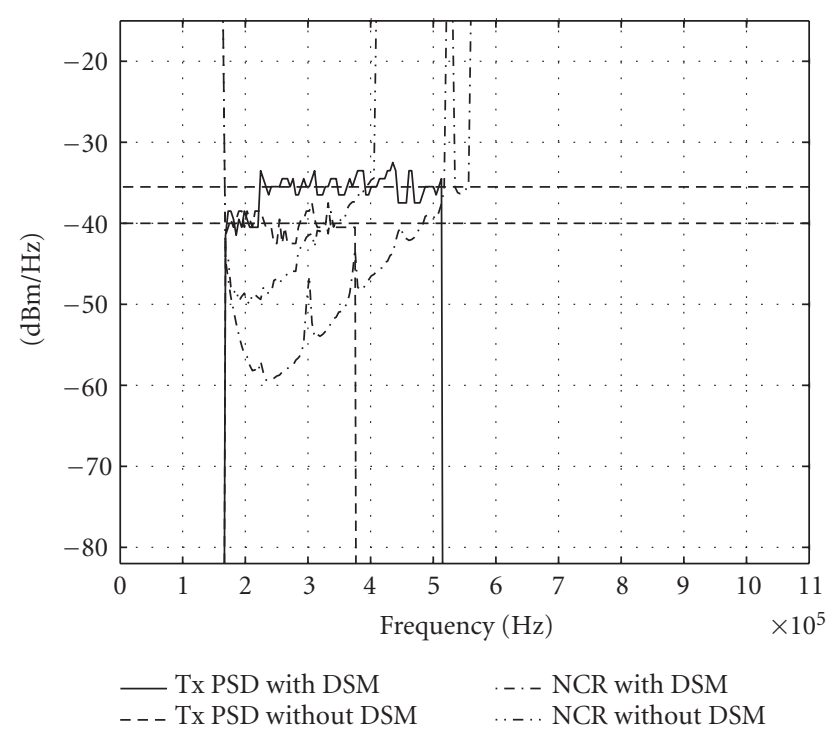

FIGURE 4: Downstream ADSL transmit power spectral density (PSD) (solid) of the ATU-C transmitting over the $5000 \mathrm{~m}$ loop, together with the noise-to-channel ratio (dotted). Average PSD with $\mathrm{DSM}=-35.6 \mathrm{dBm} / \mathrm{Hz}$ and average PSD without $\mathrm{DSM}=-40 \mathrm{dBm} / \mathrm{Hz}$.

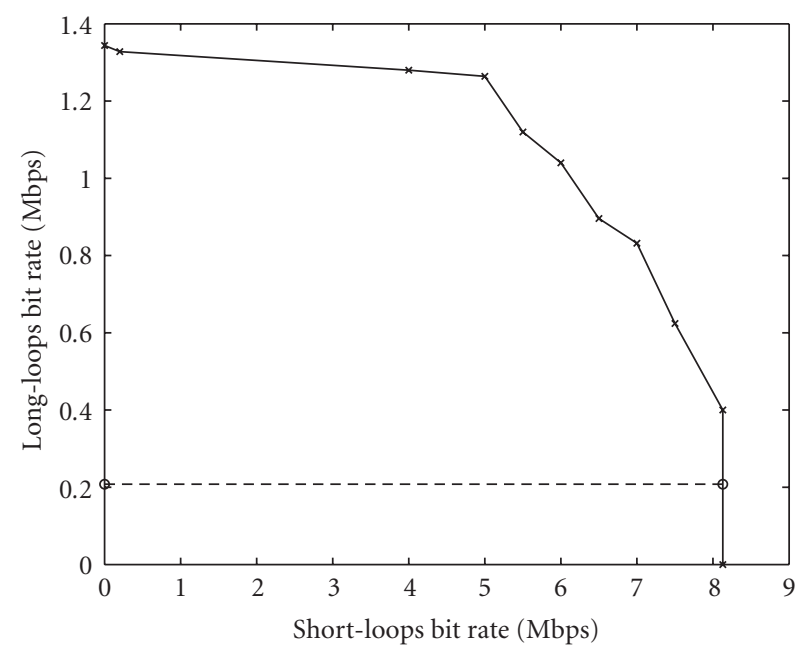

FIGURE 5: Rate region for the short- and long-loops scenario: without DSM (dotted, circles) and with DSM (solid, plusses). of central office (CO) distributed and remote terminal (RT) distributed lines in the same cable binder.

The demonstrator allows switching from normal mode to DSM mode for downstream only. DSM is only applied to the downstream PSD as the upstream does not suffer significantly from crosstalk. In DSM mode, some modem parameters are switched to ensure PA operation, and in addition the ADSL transceivers switch from a normal modem software build to a DSM modem software build. Some changes have been made to the modem software to allow DSM at level 1 , where the water-filling is approximated by a flat PSD.

The changes in the software consist of, in the first place, expanding the range of the average relative gain from initialization to showtime ${ }^{1}$ from $(0,-12) \mathrm{dB}$ to $(6,-20,5) \mathrm{dB}$. This means that a larger power back-off and boosting are made possible. A second topic of software changes concerns the

\footnotetext{
${ }^{1}$ Showtime is the state in either ATU-C or ATU-R reached after all initialization and training is completed, in which user data is transmitted [6].
} 
sync symbols in showtime. Once in showtime, the modems react to upcoming and disappearing noises coming from neighbouring lines. A modem starts up with a high noise level due to many disturbers, the transmit PSD will be calculated to achieve the SNR necessary to attain the target bit rate. If the noise then decreases due to neighbouring lines becoming inactive, the modem will automatically decrease its transmit PSD as the SNR is higher than needed. As the transmit PSD of the sync symbols may not change during showtime, it has to be low enough compared to the transmit PSD of the data symbols to avoid intersymbol interference (ISI) from the sync symbols into the data symbols. This can be either achieved by ensuring a low transmit PSD of the sync symbols or by adapting the transmit PSD of the sync symbols according to the data symbol transmit PSD variation.

The demonstrator shows a significant bit rate increase on the long CO loop. This results from, on the one hand, power back-off on the short RT loop and, on the other hand, boosting on the long CO loop. Figure 4 illustrates this boosting on the long loop. The figure shows also the noise-to-channel ratio (NCR), depicted with dotted lines.

Without DSM, only $256 \mathrm{kbps}$ is achieved on the long CO loop while the short RT loop operates at $4 \mathrm{Mps}$. With DSM, not less than $1344 \mathrm{kbps}$ is achieved on the long CO loop with still $4 \mathrm{Mbps}$ on the short RT loop. This is an increase of over $400 \%$. For a more general scenario with two long CO loops together with two short RT loops, the bit rates increase even more, namely from $208 \mathrm{kbps}$ to $1280 \mathrm{kbps}$, an increase of over $500 \%$.

Figure 5 depicts the rate region for the short and long lines with and without DSM. It is clear that DSM allows extending the rate region substantially. Remark that these results here merely indicate the potential of DSM. The results achievable in the field will depend on the actual noise environment and loop length distribution.

Although these results look very promising, iterative water-filling also has a number of drawbacks. Firstly, as shown in Figure 4, iterative water-filling results in boosting on the long loops. Boosting implies breaking the spectral mask constraints, hence spectral compatibility with other services is not assured. Spectrally compatible DSM has been investigated by means of the American spectrum management standard [3] method B compliancy [8]. Method B ensures spectral compatibility of a new technology not by imposing a spectral mask, but by ensuring that the new technology does not harm the specified basis systems. This is verified by computing the impact on, for example, the bit rate of these basis systems.

A second important drawback of iterative water-filling is the fact that DSM reduces the noise margin on the short line significantly compared to the current deployment. The lines are then operated in PA mode with $6 \mathrm{~dB}$ noise margin, which means that, if, for example, a new DSL line is activated, the short line could go out of sync due to the large noise level change. We therefore implemented a new ADSL overhead channel (AOC) message enabling the modem to request a quick $\mathrm{g}_{\mathrm{i}}$ boost $(\mathrm{QB})$. This quick $\mathrm{g}_{\mathrm{i}}$ boost message is a very short message from the $\mathrm{Rx}$ modem to the Tx modem asking for an increase in PSD on all active tones. It makes it possible for the modems to react quickly to rapidly increasing noises such as a new upcoming disturber. The short length of the message decreases the probability of corrupt reception [9], and as such enhances the stability. The nonstationary noiserobustness results are detailed in the next section.

\section{NONSTATIONARY NOISE ROBUSTNESS}

Robustness of a DSL modem against nonstationary noise translates to stability on the level of the DSL link and higher protocol layer communication links. Hence, a good robustness is a key to the development of a stable network and satisfied customers.

In this section, nonstationary noise robustness is investigated by injecting time-varying noise on the line. To show DSM gains, one typically needs multiple active DSL lines in a binder, but for the sake of simplicity, only one DSL line is taken into account here and the nonstationary noise is emulated. As DSM is only applied to downstream transmission in the case of ADSL, the noise injection happens only at the customer premises equipment (CPE) side. Many parameters play a role in the noise-robustness measurement: loop length, bit rate, noise margin, injected noise level, noise level change, and so forth, but, as can be seen in the next section, the results show that the key parameters are the noise margin, power back-off, changing noise level, and number of active tones. Indeed, the nonstationary noise robustness is by definition the robustness against the changing noise level. However, the study will also show that the level of power back-off influences the results. In this study, the spectral shape of the noise has been kept flat over the entire bandwidth.

\subsection{Noise-robustness measurements}

DSM, that is, PA mode of operation, is achieved by provisioning the modems with a target bit rate and a maximum additional noise margin set to zero. The target noise margin is set to $6 \mathrm{~dB}$ and the only noise robustness the modems have left beside this noise margin is the bit swap procedure. Unfortunately, the bit swap protocol is limited to maximum 6 swaps per message [6]. Furthermore, the bit swap is done over the ADSL overhead channel (AOC) with at least 800 milliseconds between every two bit swap messages. Both restrictions limit the achievable noise-increase recovery. The measurement results for DSM, when all tones are loaded with bits, are shown in Figure 6 and labelled as "DSM without QB." The label "DSM with QB" is explained further. The modems are DSM-enabled prototypes and can apply power back-off up to $20.5 \mathrm{~dB}$, in comparison with conventional ADSL1 modems, which are limited to a $12 \mathrm{~dB}$ power back-off. The figure shows the maximum noise increase an ADSL transceiver can handle without resynchronization versus the power back-off level.

Figure 6 shows us that conventional ADSL1 modems operating at fixed margin (DSM without QB) can only recover from a maximum noise increase of $7.5 \mathrm{~dB}$. Indeed, the maximum power back-off for a conventional ADSL1 modem is 


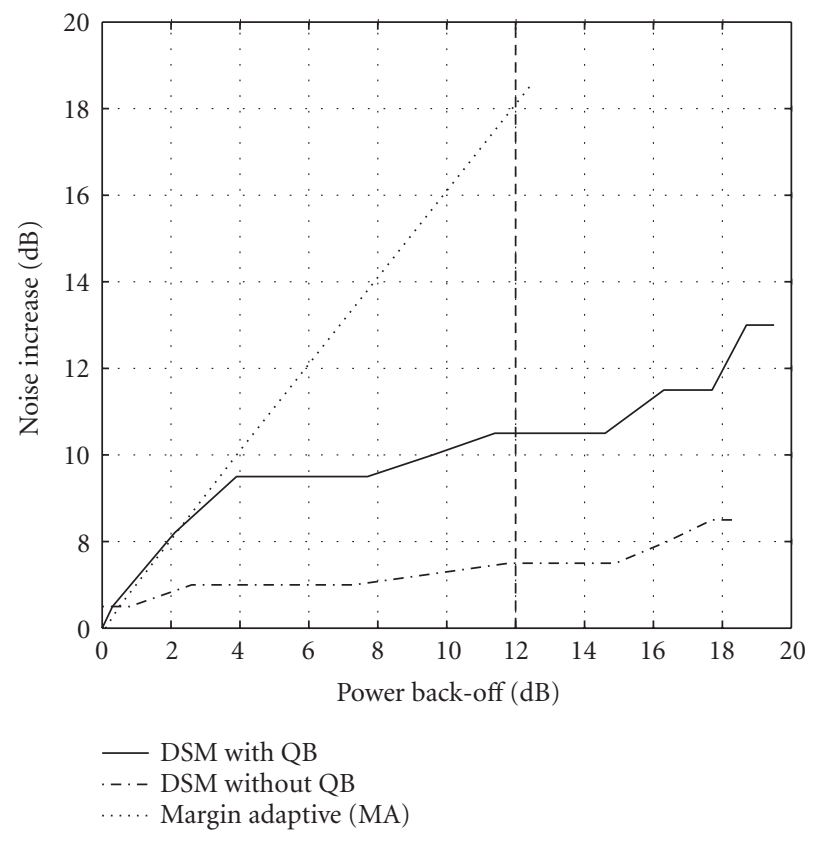

FIgURE 6: Noise-robustness measurements with all tones active.

limited to $12 \mathrm{~dB}$, for which the figure shows that the maximum noise increase is equal to $7.5 \mathrm{~dB}$. If the power back-off can go up to $20.5 \mathrm{~dB}$, a better noise robustness is obtained up to $13 \mathrm{~dB}$. The explanation can be found in the difference between initialization and showtime. During initialization, the modem is training and transmits at $-40 \mathrm{dBm} / \mathrm{Hz}$ (somewhat lower if politeness is applied). During the training period, a noise measurement is performed on which the modem will compute the power back-off value assuming that the noise level will remain constant. The noise measurement not only comprises the background noise and crosstalk noise, but also signal-related noises such as intersymbol interference (ISI), intercarrier interference (ICI), and noise inherent to the modem. This means that if a modem performs a large power back-off, the total noise will decrease also. This is why the noise margin, when entering showtime, is slightly larger than $6 \mathrm{~dB}$ and increasing with increasing power back-off, which thus results in a slightly better noise robustness.

The figure also shows the comparison with marginadaptive operation, which is equivalent to no DSM. In this case, the $x$-axis has to be seen as additional noise margin instead of power back-off. Indeed, the noise margin is then not decreased as no power back-off is applied. As expected, the robustness against a sudden noise increase grows linearly with the noise margin.

In case only a few tones are loaded with bits, the modems operating in DSM mode perform better against nonstationary noise. Indeed, with only few active tones, the bit swap can increase the transmit PSD faster than when many tones are active. The results when 40 tones are active are shown in Figure 7. However, this study focuses on high bit rates with modems applying power back-off. Modems with only a few active tones are most of the time operated at the line's

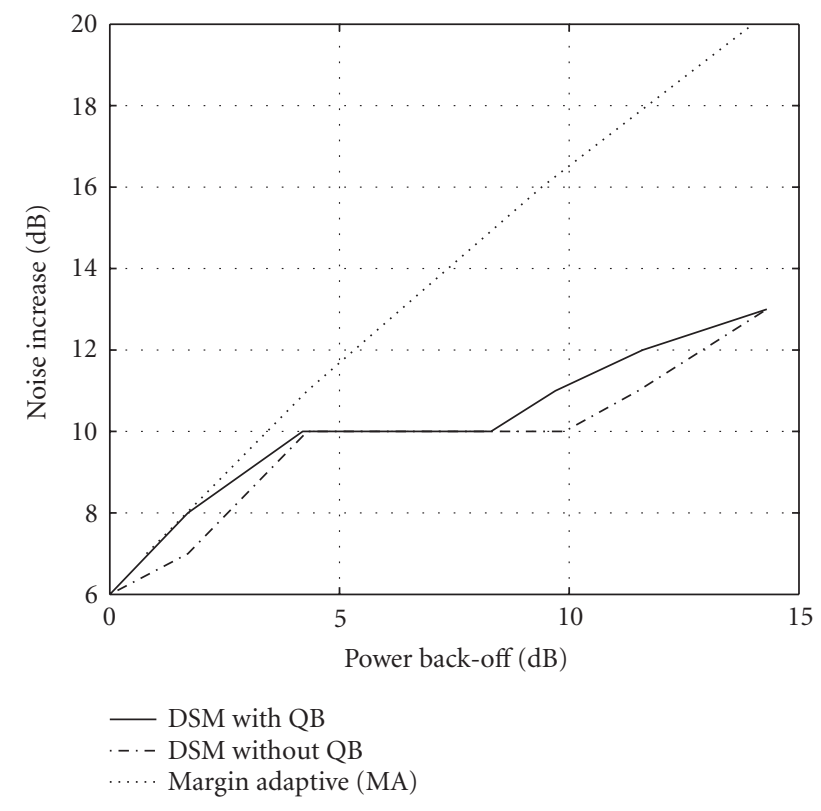

Figure 7: Noise-robustness measurement on long loop (40 tones active).

maximum bit rate, be it a low bit rate, and hence no power back-off can be applied.

\subsection{Improved noise robustness with quick $\mathrm{g}_{\mathrm{i}}$ boost}

Applying DSM with a slow bit swap algorithm makes it impossible for the modem to adapt to quick noise or channel changes. Therefore, we have introduced a quick $\mathrm{g}_{\mathrm{i}}$ boost message, that is, a very short message from the $\mathrm{Rx}$ to the Tx modem asking to boost all carriers with a certain gain included in the message. The noise robustness increases thanks to two factors. First, the message is very short. This lowers the probability of corrupt message reception [9]. Second, the transmit PSD of all carriers is increased at once. In our experimental setup, the noise increase is flat, for which a flat quick $\mathrm{g}_{\mathrm{i}}$ boost gives great benefit. But even for shaped noise increases, a quick $\mathrm{g}_{\mathrm{i}}$ boost makes it possible to recover very fast from a negative noise margin. The finetuning to restore the noise margin to the same level for all carriers happens then with the traditional bit swap mechanism.

In Figures 6 and 7, the possible noise increases versus power back-off is denoted as 'DSM with QB'. As can be seen from the figure, the noise robustness is better by up to $3 \mathrm{~dB}$ compared to DSM without quick $g_{i}$ boost. DSM with quick $g_{i}$ boost can be said to be as stable as fixed power operation up to $4 \mathrm{~dB}$ power back-off. So a modem with the quick $\mathrm{g}_{\mathrm{i}}$ boost can easily apply $4 \mathrm{~dB}$ power back-off: if extra noise forces the modem to increase its power, then the quick $\mathrm{g}_{\mathrm{i}}$ boost is fast enough such that this line has the same noise robustness as a line that does not apply power back-off. Once more power back-off is applied, the noise robustness decreases. This means that there is clearly a tradeoff between the performance gains of applying power back-off (as indicated in 


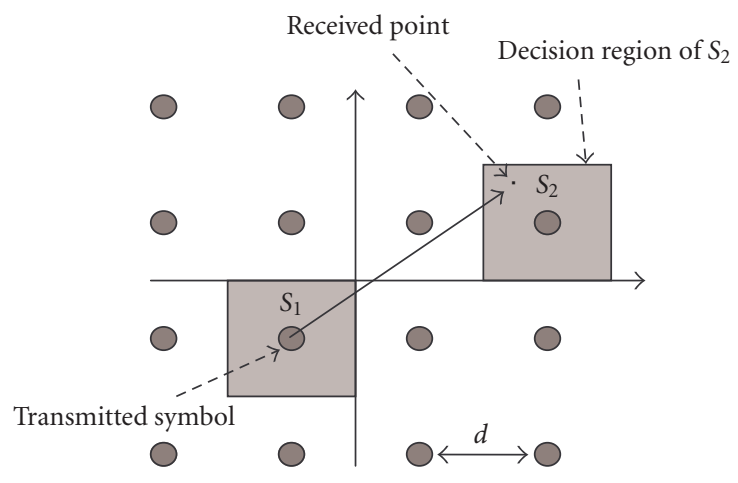

FIGURE 8: QAM hard-decision symbol detection and its impact on noise measurement.

Section 4) and the robustness. A quick $\mathrm{g}_{\mathrm{i}}$ boost can improve the robustness (with $4 \mathrm{~dB}$ ) of a system applying power backoff, but there is still a difference in robustness between a system applying power back-off and a system that does not apply power back-off at all (MA mode of operation).

There are 2 reasons for this relatively small robustness when DSM is applied. First, the noise measurement within the modem is not accurate when large noise increases occur. Indeed, QAM demodulation at the receiver is based on hard decisions and the process of corrupt symbol detection is shown in Figure 8. After the decision is made, right or wrong, the difference between the demodulated symbol and the received signal is measured as noise. This means that the measured noise will never exceed the maximum distance from demodulated to received symbol within one decision region, which is $(\sqrt{2} / 2) \cdot d$. As a consequence, the modem measures a noise increase smaller than it is actually, and therefore makes a request for a quick $g_{i}$ boost that is too small compared to the noise.

Second, the AOC protocol carrying the bit swap is slow, such that no more than two consecutive quick $g_{i}$ boost messages can be carried out before the modem goes out of showtime. It has to be noted that AOC and bit swap are improved in ADSL2, but still no quick $\mathrm{g}_{\mathrm{i}}$ boost message is implemented.

No investigation has been done on mutual interference and stability of several modems applying quick $g_{i}$ boosts. Although the convergence of iterative water-filling has been proven theoretically in [2], the stability of many modems applying DSM together with quick $\mathrm{g}_{\mathrm{i}}$ boosts is still an open issue. Indeed, a quick $\mathrm{g}_{\mathrm{i}}$ boost changes suddenly the transmit PSD on one line, but this results in a sudden change of the crosstalk seen by neighbouring lines, hence potentially triggering a quick $\mathrm{g}_{\mathrm{i}}$ boost on the neighbouring lines.

\section{CONCLUSION}

In this paper, the practical implementation of distributed multiuser power allocation, more specifically iterative waterfilling, is investigated together with its performance on the cable farm at Alcatel Research and Innovation Labs. We show that when water-filling is applied to discrete multitone systems, it is possible to approximate the obtained PSDs by flat transmit spectra, hence significantly reducing the complexity of the power allocation algorithm.

The results of iterative water-filling implemented on ADSL modems show a significant performance increase compared to the deployment mode currently used by the operators (margin-adaptive mode). The rate region is plotted for a particular deployment case showing the huge advantages of iterative water-filling.

As a consequence of the overall bit-rate optimization, some DSL lines decrease their transmit power to mitigate the induced interference on neighbouring lines. Hence, the noise margin is decreased to improve the performance.

As the noise margin acts as a protection against nonstationary noise, we investigated the impact of DSM on nonstationary noise robustness. The results show clearly that DSM trades off nonstationary noise robustness for performance.

Finally, a new scheme is proposed to improve the nonstationary noise robustness when applying DSM. It speeds up the ADSL overhead channel by requesting a quick $\mathrm{g}_{\mathrm{i}}$ boost, and therefore improves the nonstationary noise robustness with approximately $3 \mathrm{~dB}$.

\section{ACKNOWLEDGMENTS}

This work was supported in part by the IWT MIDAS Project (030032), Multistandard integrated devices for broadband DSL access and in-home powerline communications. Part of this work has been presented at the IEEE International Conference on Acoustics, Speech, and Signal Processing (ICASSP), May 2004 and the IASTED Communication Systems and Networks Conference (CSN), September 2004.

\section{REFERENCES}

[1] T. Starr, M. Sorbara, J. M. Cioffi, and P. J. Silverman, DSL Advances, Prentice Hall Communications Engineering and Emerging Technologies Series, Prentice Hall PTR, Upper Saddle River, NJ, USA, 2002.

[2] W. Yu, G. Ginis, and J. M. Cioffi, "Distributed multiuser power control for digital subscriber lines," IEEE Journal on Selected Areas in Communications, vol. 20, no. 5, pp. 1105-1115, 2002.

[3] "Spectrum management for loop transmission systems, Issue 2,” ANSI, ANSI-T1.417, November 2002.

[4] K. J. Kerpez, D. L. Waring, S. Galli, J. Dixon, and P. Madon, "Advanced DSL management," IEEE Communications Magazine, vol. 41, no. 9, pp. 116-123, 2003.

[5] G. Ginis and J. M. Cioffi, "Vectored transmission for digital subscriber line systems," IEEE Journal on Selected Areas in Communications, vol. 20, no. 5, pp. 1085-1104, 2002.

[6] "Asymmetrical digital subscriber line (ADSL) transceivers," ITU Std G.992.1, 1999.

[7] "Asymmetrical digital subscriber line (ADSL) transceivers," ITU Std G.992.1, 2003.

[8] R. Suciu, E. Van den Bogaert, J. Verlinden, and T. Bostoen, "Ensuring spectral compatibility of DSM," in Proceedings of EURASIP 12th European Signal Processing Conference (EUSIPCO '04), Vienna, Austria, September 2004. 
[9] J. Verlinden, E. Van den Bogaert, T. Bostoen, R. Cendrillon, and M. Moonen, "Protecting the robustness of ADSL and VDSL DMT modems when applying DSM," in Proceedings of IEEE International Zurich Seminar on Communications (IZS '04), pp. 140-143, Zurich, Switzerland, February 2004.

Etienne Van den Bogaert is a DSL Research Engineer at the Research \& Innovation Department in Antwerp, Belgium. In this function, he is investigating nextgeneration DSL technologies and their applications. His current main research interest is dynamic spectrum management (DSM): algorithms, impact on performance and stability, and practical implementation.

Tom Bostoen received the M.S. degree in physical engineering from Ghent University, Ghent, Belgium, in 1998. Tom Bostoen is currently the Product Manager of the 5530 network analyzer at the Access Networks Division of Alcatel in Antwerp, Belgium. In his previous function, he was the Project Manager of the DSL Physical Layer Research Project at the Research \& Innovation Department. Before that, he studied single-ended line testing (SELT) as a Research Engineer in the same department and contributed to ITU G.selt standardization.

Jan Verlinden received a degree in electrical engineering in 2000 from the KULeuven, Belgium. He is currently Member of the DSL Experts Team of the Access Networks Division in Antwerp, Belgium. He joined the Research \& Innovation Division of Alcatel in September 2000, where he focussed on echo canceller techniques. From 2002 on, he has focussed on DSM. As such, he participated in the VDSL Olympics by
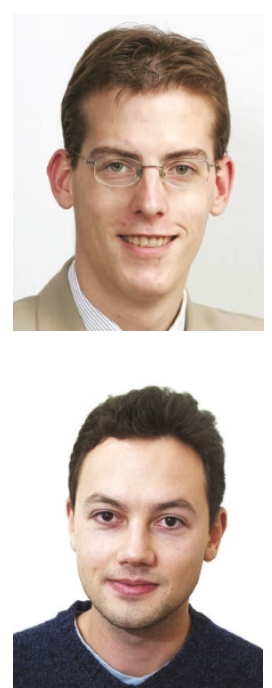
introducing DSM into the VDSL prototype. Within the DSL Experts Team, he is currently studying emerging DSL physical layer technologies. He also contributes to ANSI NIPP-NAI standardization.

Raphael Cendrillon was born in Melbourne, Australia, in 1978. He received an Electrical Engineering degree (first class honours) from the University of Queensland, Australia, in 1999, and a Ph.D. degree in electrical engineering at the Katholieke Universiteit Leuven, Belgium in 2004. His Ph.D. was awarded summa cum laude with congratulations of the jury, an honor given to the top $5 \%$ of Ph.D. graduates. His re-

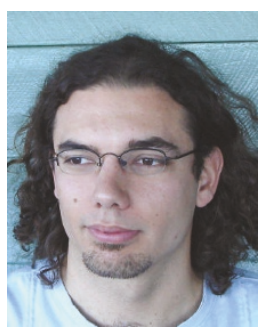
search focuses on the application of multiuser communication theory to xDSL. In 2002, he was a Visiting Scholar at the Information Systems Laboratory, Stanford University with Professor John Cioffi. Since 2005, Dr. Cendrillon has been a PostDoctoral Research Fellow at the University of Queensland, Australia. His work in xDSL is done in close collaboration with Alcatel Research \& Innovation Department, Belgium, for which he was awarded the Alcatel Bell Scientific Prize in 2004. He was also the recipient of an IEEE Travel Grant in 2003 and 2004, and the KU Leuven Bursary for Advanced Foreign Scholars in 2004.
Marc Moonen received the Electrical Engineering degree and the Ph.D. degree in applied sciences from Katholieke Universiteit Leuven, Leuven, Belgium, in 1986 and 1990, respectively. Since 2004, he is a Full Professor at the Electrical Engineering Department of Katholieke Universiteit Leuven, where he is currently heading a research team of 16 Ph.D. candidates and postdocs, working in the area of signal processing for

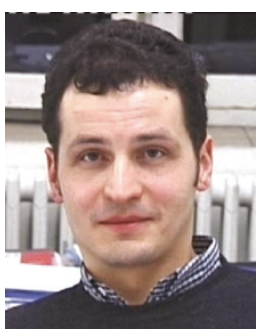
digital communications, wireless communications, DSL, and audio signal processing. He received the 1994 KU Leuven Research Council Award, the 1997 Alcatel Bell (Belgium) Award (with Piet Vandaele), the 2004 Alcatel Bell (Belgium) Award (with Raphael Cendrillon), and was a 1997 'Laureate of the Belgium Royal Academy of Science'. He was the Chairman of the IEEE Benelux Signal Processing Chapter (1998-2002), and is currently a EURASIP AdCom Member (European Association for Signal, Speech and Image Processing, from 2000 up till now. He has been a Member of the editorial board of IEEE Transactions on Circuits and Systems II (20022003). He is currently Editor-in-Chief for the EURASIP Journal on Applied Signal Processing from 2003 up till now, and a Member of the editorial board of Integration, the VLSI Journal, EURASIP Journal on Wireless Communications and Networking, and IEEE Signal Processing Magazine. 